Dilip R. Karnad

Rakesh Bhadade

Pradeep K. Verma

Nivedita D. Moulick

Mradul K. Daga

Neelima D. Chafekar

Shivakumar Iyer

\section{Intravenous administration of ulinastatin (human urinary trypsin inhibitor) in severe sepsis: a multicenter randomized controlled study}

Received: 29 December 2013

Accepted: 24 March 2014

Published online: 16 April 2014

(C) The Author(s) 2014. This article is published with open access at

Springerlink.com

Take-home message: Ulinastatin, a protease inhibitor, inhibits several proinflammatory proteases and decreases inflammatory cytokine levels and mortality in experimental sepsis. In this pilot study, intravenous administration of ulinastatin (200,000 IU 12 hourly for 5 days) reduced mortality, new onset of organ dysfunction, duration of mechanical ventilation and hospital stay in patients with severe sepsis when started within $48 \mathrm{~h}$ of onset of failure of one or more organs.

Electronic supplementary material The online version of this article (doi: 10.1007/s00134-014-3278-8) contains supplementary material, which is available to authorized users.

\section{R. Karnad}

Critical Care, Jupiter Hospital, Thane, India

R. Bhadade

Department of Medicine, B Y L Nair Hospital and $\mathrm{T} \mathrm{N}$ Medical College, Mumbai, India

P. K. Verma

Department of Anesthesia, Vardhaman Mahavir Medical College and Safdarjung Hospital, New Delhi, India

\section{N. D. Moulick}

Department of Medicine, Lokmanya Tilak Muncipal General Hospital and Medical College, Mumbai, India
M. K. Daga

Department of Medicine, Maulana Azad Medical College, New Delhi, India

N. D. Chafekar

Department of Medicine, NDMVP Samaj Medical College, Nashik, India

\section{S. Iyer}

Jehangir Clinical Development Centre,

Pune, India

D. R. Karnad (

10, Ramashree, Hindu Colony Road No 1, Dadar (East), Mumbai 400014, India e-mail: drkarnad@ rediffmail.com

Tel.: +91-9892114002

Abstract Purpose: Ulinastatin, a serine protease inhibitor, inhibits several pro-inflammatory proteases and decreases inflammatory cytokine levels and mortality in experimental sepsis. We studied the effect of ulinastatin on 28-day all-cause mortality in a double-blind trial in patients with severe sepsis in seven Indian hospitals. Methods: Patients with sepsis were randomized within $48 \mathrm{~h}$ of onset of one or more organ failures to receive intravenous administration of ulinastatin (200,000 IU) or placebo 12 hourly for 5 days. Results: Of 122 randomized subjects, 114 completed the study ( 55 receiving ulinastatin, 59 receiving placebo). At baseline, the mean APACHE II score was $13.4(\mathrm{SD}=4.4), 48(42 \%)$ patients were receiving mechanical ventilation, $58(51 \%)$ were on vasopressors, and $35 \%$ had multiple organ failure. In the modified intention-to-treat analysis (patients receiving six or more doses of study drugs), 28-day all-cause mortality was $7.3 \%$ with ulinastatin (4 deaths) versus $20.3 \%$ (12 deaths) with placebo $(p=0.045)$. On multivariate analysis too, treatment with ulinastatin (odds ratio $0.26,95 \% \mathrm{CI}$ $0.07-0.95 ; p=0.042$ ) independently decreased 28-day all-cause mortality. However, the mortality difference did not reach statistical significance in the intention-to-treat analysis [10.2 \% (6/ 59 deaths) with ulinastatin versus $20.6 \%$ (13/63 deaths) in the placebo group; $p=0.11]$. The ulinastatin group had lower incidence of newonset organ failure (10 vs. 26 patients, $p=0.003)$, more ventilator-free days (mean \pm SD $19.4 \pm 10.6$ days vs. $10.2 \pm 12.5$ days, $p=0.019$ ), and shorter hospital stay $(11.8 \pm 7.1$ days vs. $24.2 \pm 7.2$ days, $p<0.001)$. Conclusions: In this pilot study, intravenous administration of ulinastatin reduced mortality in patients with severe sepsis in the modified intention-to-treat analysis, but not in the intention-to-treat analysis.

Keywords Serine protease inhibitor . Inflammatory mediators .

Bacterial infection - Septic shock . Pneumonia $\cdot$ Abdominal sepsis 


\section{Introduction}

Sepsis is a common cause of morbidity and mortality in critically ill patients, and its incidence is increasing worldwide annually $[1,2]$. The pathogenesis of sepsis is complex and is believed to be initiated by the interaction between pathogen-associated molecular patterns and pattern recognition receptors on host immune cells [3, 4]. This sets off a series of pro-inflammatory mechanisms including synthesis and release of cytokines and complement, chemotaxis and activation of neutrophils, and initiation of coagulation [3-5]. These, in turn, have widespread effects on other cells including inflammatory cells, immune response, endocrine and autonomic nervous systems, and vascular endothelium, mainly aimed at limiting spread or eliminating the infecting pathogen [36]. Current opinion suggests that the systemic inflammatory response syndrome (SIRS) that characterizes severe sepsis results from an excessive activation of proinflammatory mediators, which have pleiotropic effects that overwhelm the body's anti-inflammatory mechanisms, leading to widespread vascular, endothelial, and organ dysfunction that is often fatal [3-6].

Many of the intermediaries in the systemic inflammatory processes are serine proteases. These include trypsin, thrombin, chymotrypsin, kallikrein, plasmin, neutrophil elastase, cathepsin, neutrophil protease-3, and coagulation factors IXa, Xa, XIa, and XIIa [7, 8]. It is now being recognized that besides their proteolytic activity, these proteases have an important role in regulation of inflammation through inter- and intracellular signaling pathways $[8,9]$. To counter-regulate the effect of these proteases, several protease inhibitors are produced by the liver in the presence of inflammation; these include acute phase reactants such as $\alpha_{1}$-antitrypsin and proteins of the inter- $\alpha$-inhibitor family [9]. Urinary trypsin inhibitor is one such important protease inhibitor found in human blood and urine; it has been also referred to in the literature as ulinastatin or bikunin [10,11]. It is an acidic glycoprotein (molecular weight $30 \mathrm{kDa}$ ) and Kunitz-type serine protease inhibitor composed of 143 amino acid residues and includes two Kunitz-type domains [7, 10]. It is cleaved from the larger inter- $\alpha$-trypsin inhibitor molecule by neutrophil elastase in the presence of inflammation, and is believed to play an important antiinflammatory role [8-12].

Studies in patients have shown that there is a decrease in serum levels of ulinastatin in sepsis, with the lowest levels being found in patients with severe sepsis and septic shock [12]. As compared to wild-type mice, mortality due to experimental sepsis is higher in genetically modified knockout mice that lack the genes for synthesis of urinary trypsin inhibitor $[11,13]$. Several preclinical studies have shown a reduction in the systemic inflammatory response and organ dysfunction due to sepsis in animals treated with ulinastatin [13-17]. On the basis of a few small clinical studies that have shown a trend towards reduced mortality and duration of hospitalization with ulinastatin in severe sepsis [18, 19], some authors have suggested that ulinastatin may have a role as a novel therapy in severe sepsis $[20,21]$. We therefore conducted this pilot study to evaluate the efficacy of ulinastatin in Indian patients with severe sepsis.

\section{Methods}

This randomized, double-blind, placebo-controlled trial was conducted in the intensive care units (ICUs) of seven tertiary care hospitals in India; the study protocol was approved by the institutional review boards at each center. The study protocol is available from the Indian clinical trials registry at http://www.ctri.nic.in (clinical trial number CTRI/2009/091/000650).

Adults, aged 18-60 years (both inclusive), with severe sepsis, admitted to the ICU between September 2009 and June 2010 were eligible for enrollment into the study. Sepsis was defined as evidence of infection (defined as presence of white blood cells in a normally sterile body fluid, perforated abdominal viscus, evidence of pneumonia, or presence of a condition associated with a high risk of bacterial infection, e.g., ascending cholangitis), and the presence of at least three of the four SIRS criteria [22]. Severe sepsis was defined as the presence of sepsis as defined above, along with dysfunction of at least one organ or system. Cardiovascular system failure was defined as systolic blood pressure $\leq 90 \mathrm{mmHg}$ or mean arterial pressure $\leq 70 \mathrm{mmHg}$ for at least $1 \mathrm{~h}$ despite adequate fluid resuscitation, or the use of vasopressors to maintain arterial pressure above these levels or unexplained metabolic acidosis $(\mathrm{pH} \leq 7.30$ or base deficit $\geq 5.0 \mathrm{mmol} / \mathrm{L}$ ) with plasma lactate greater than 1.5 times the upper limit of normal. Renal failure was defined as urine output $<0.5 \mathrm{~mL} / \mathrm{kg} / \mathrm{h}$ for $1 \mathrm{~h}$, or serum creatinine levels greater than 2.5 times the upper limit; respiratory failure as $\mathrm{PaO}_{2} / \mathrm{FiO}_{2} \leq 250$ in the presence of other dysfunctional organs or $\leq 200$ if only lung; hematologic dysfunction by platelet count $<80,000 / \mathrm{mm}^{3}$ or $50 \%$ drop in preceding 3 days [22]. Only patients with organ dysfunction of $\leq 48 \mathrm{~h}$ duration were eligible for inclusion.

Pregnant or breastfeeding women, patients with platelet count $<30,000 / \mathrm{mm}^{3}$, history of organ transplantation, poorly controlled neoplasm, end-stage chronic kidney or liver disease, and patients weighing $>135 \mathrm{~kg}$ were excluded. Patients in whom limitation of care was planned or who were expected to die within the next $24 \mathrm{~h}$ were also excluded.

Block randomization (block size of 4) using a computer-generated sequence was used to ensure balance 
between treatments; blinding of the sequence was maintained in sealed, opaque envelopes. Blinding of treatment allocation in participating centers was done using serially numbered packages of the study medication; package numbered 1 was to be used for the first patient, and so on. After obtaining written informed consent, patients were randomized in a 1:1 ratio to receive ulinastatin or placebo in addition to standard care. Randomized patients received an intravenous infusion of either 200,000 IU ulinastatin (U-tryp, Bharat Serum and Vaccines Ltd, India) or identical placebo dissolved in $250 \mathrm{~mL}$ of $0.9 \%$ saline given intravenously over $1 \mathrm{~h}$ every $12 \mathrm{~h}$ for 5 days in a double-blind evaluation. For patients with fluid restriction, $100 \mathrm{~mL}$ of $0.9 \%$ saline could be used. Infusion could be interrupted for 1 day, if there was greater than three times increase in liver enzymes over baseline levels.

In addition to the study medications, patients also received antibiotics, intravenous fluids, enteral or parenteral nutrition, transfusion of blood and blood products, and supportive care for organ dysfunction including mechanical or non-invasive ventilation, vasopressors (noradrenaline, adrenaline, dopamine, or vasopressin), or dialysis as per the standard treatment protocols followed in each ICU. Investigators were encouraged to follow the Surviving Sepsis Campaign guidelines. No concomitant medications were prohibited in the study.

Baseline characteristics including demographics, preexisting conditions, organ dysfunction, infection, and hematologic and other laboratory tests were assessed within the $24 \mathrm{~h}$ prior to infusion of the first dose of study medication. Blood samples were also obtained on the day of discharge. Patients were followed up till 28 days after the start of treatment. The primary end-point for this study was 28-day all-cause mortality. Secondary end-points included onset of new organ failure, duration of vasopressor use, ventilator-free days till day 28 , and length of hospital stay. New-onset organ failure was defined as occurrence of organ failure after randomization. The definitions used were the same as those used in inclusion criteria; liver dysfunction was defined as serum bilirubin $\geq 2.0 \mathrm{mg} / \mathrm{dL}$ when bilirubin was $<2.0 \mathrm{mg} / \mathrm{dL}$ at baseline and central nervous system failure was defined as Glasgow coma scale (GCS) $\leq 10$ after randomization in patients who had baseline GCS $>10$.

\section{Statistical analysis}

Data analysis was carried out using the Graph Pad Instat and SPSS version 14 software package. Forward stepwise multiple logistic regression analysis was used for assessment of the primary end-point. Other categorical data were compared between the treatment groups by Pearson's Chi-squared test or Fisher's exact test, as appropriate. The unpaired $t$ test was used for continuous variables and the Mann-Whitney test for ordinal data like the APACHE II score.

Sample size calculation was performed assuming a 28-day all-cause mortality of $30 \%$ in the control group and $10 \%$ in the study group. A sample size of 59 completed patients in each group was required to attain a power of $80 \%$ at a significance level of $5 \%$. The primary efficacy end-point of 28-day all-cause mortality was assessed in the modified intention-to-treat population which was defined a priori. This primary analysis population included all randomized patients who had received at least six doses of the study medication. Safety was assessed in the safety population, defined as all subjects who received even a single dose of the study medication. Mortality difference in the intention-to-treat population that included all randomized subjects was analyzed as a secondary end-point.

\section{Results}

During the 10-month study period, 224 patients met the inclusion criteria. Of these, 102 patients were excluded; 99 because of presence of one or more exclusion criteria, or refusal of consent to participate in the study and three patients were not enrolled as they were expected to die within $24 \mathrm{~h}$ (Fig. 1). Of the 122 randomized subjects, 114 completed the study (55 subjects in the ulinastatin group and 59 subjects in the control group). Five subjects discontinued from the study (three subjects withdrew consent and two due to protocol violation). Three patients who died within $48 \mathrm{~h}$ of enrollment were not included for analysis in the modified intention-to-treat population. Of the 114 patients who completed the study, one patient in the ulinastatin group received six doses and one patient each in the placebo group received six and seven doses; all others received the 10 scheduled doses of study medications.

\section{Baseline characteristics}

At baseline, the two treatment groups were similar with respect to demographic characteristics, cause of sepsis, number of organs affected, pattern of organ dysfunction, and need for vasopressors or mechanical ventilation (Table 1). Infection was microbiologically confirmed in 26 patients $(23 \%)$. Approximately $35 \%$ of patients had multiple organ failure at baseline, $51 \%$ required vasopressors, and $42 \%$ received mechanical ventilation. For details of organisms causing sepsis and antibiotics used, see Electronic Supplementary Material. 
Fig. 1 CONSORT diagram of the study

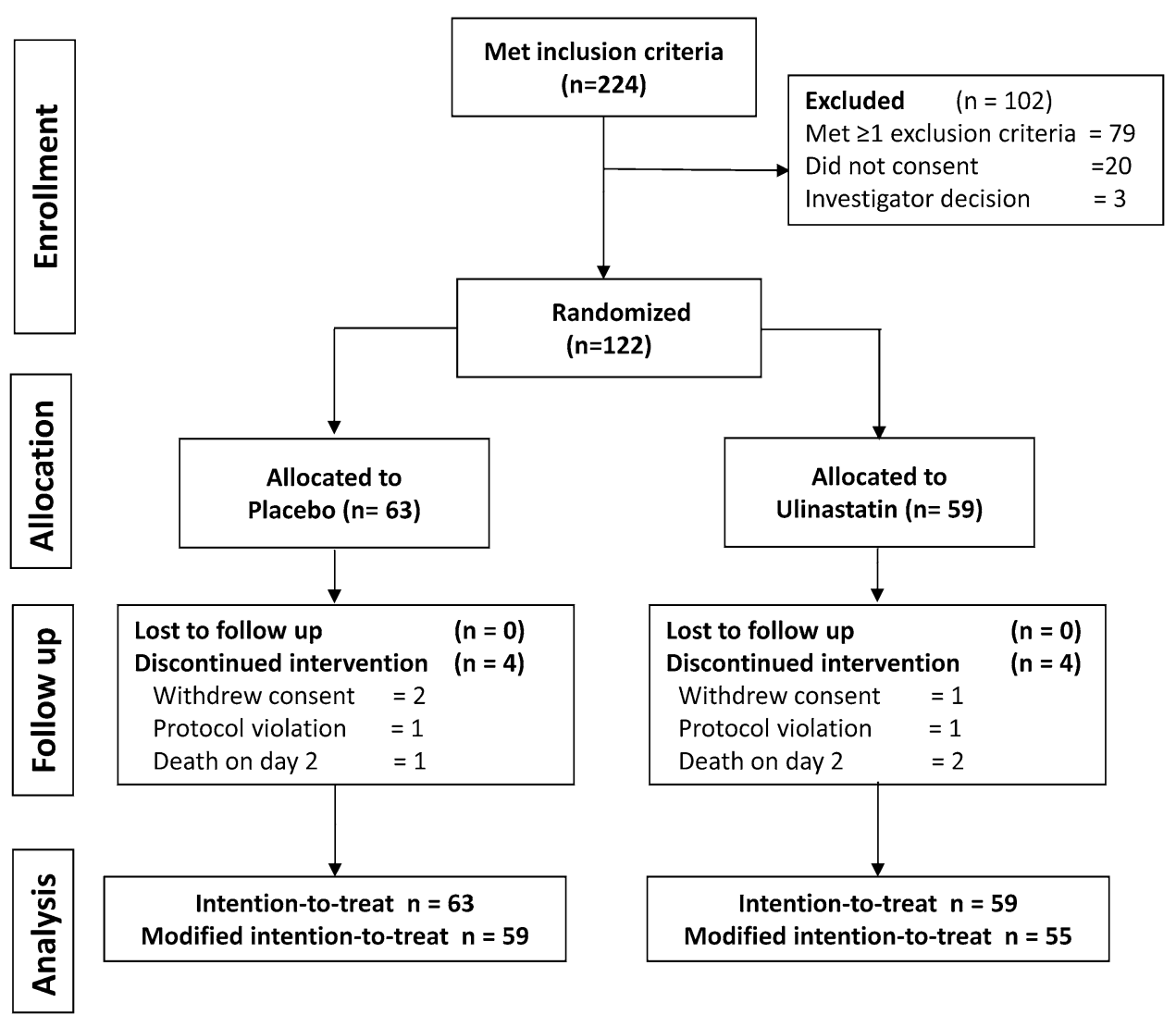

\section{Outcomes}

The 28-day mortality, the primary end-point of this study, showed a significant difference (Table 2) in the two treatment groups; there were four deaths $(7.3 \%)$ in the ulinastatin group versus 12 deaths $(20.3 \%)$ in the placebo group. On stepwise multiple logistic regression, treatment with ulinastatin was found to produce a statistically significant decrease in risk of death (odds ratio 0.26, $95 \%$ confidence limits $0.07-0.95 ; p=0.042$ ). Other variables independently associated with mortality were acute renal failure and mechanical ventilation at baseline (Table 3). Of the four deaths in the ulinastatin group one patient each died as a result of ARDS, acute renal failure, refractory shock, and multiple organ failure. Of the 12 deaths in the placebo group five patients died as a result of ARDS, five as a result of refractory shock, and one each as a result of intracranial hemorrhage and multiple organ failure. All deaths were attributed by the investigators to progression of underlying sepsis. The Kaplan-Meier plot for survival in the two groups is shown in Fig. 2.

Among the secondary end-points, there were 6 deaths $(10.2 \%)$ in the ulinastatin group $(n=59)$ in the intention-to-treat population which included all randomized subjects versus 13 deaths $(20.6 \%)$ in the control group $(n=63 ; p=0.11)$. New onset of organ dysfunction was seen in 10 subjects in the ulinastatin group and 26 subjects in the placebo group $(p=0.003)$. Number of ventilator-free days up to day 28 (mean \pm SD) was significantly more $(19.4 \pm 10.6$ days $)$ in the ulinastatin group and $10.2 \pm 12.5$ days in the placebo group $(p=0.019)$. Mean hospital stay too was less in the ulinastatin group by an average of 12.4 days $(p=0.001)$.

\section{Complications}

Intracranial hemorrhage was the only unexpected serious adverse event in one patient; this patient was from the placebo group. There were no infusion-related adverse events associated with treatment with ulinastatin.

\section{Discussion}

This prospective, double-blind, randomized, placebocontrolled trial of ulinastatin in patients with severe sepsis showed that intravenous administration of ulinastatin in a dose of 200,000 units twice daily for 5 days was associated with a reduction in 28-day all-cause mortality (the primary end-point) to 7.3 versus $20.3 \%$ in the placebo control group. A few small studies, published in Chineselanguage journals, have shown lower mortality in patients 
Table 1 Baseline characteristics in patients with severe sepsis randomized to placebo and ulinastatin treatment groups

\begin{tabular}{|c|c|c|c|}
\hline Characteristics & Placebo group $(n=59)$ & Ulinastatin group $(n=55)$ & $p$ value \\
\hline Age $(\text { years })^{b}$ & $36.7 \pm 12.5$ & $37.5 \pm 12.9$ & 0.70 \\
\hline \multicolumn{4}{|l|}{ Gender } \\
\hline Males & $50(85 \%)$ & $38(69 \%)$ & \multirow[t]{2}{*}{0.05} \\
\hline Females & $9(15 \%)$ & $17(31 \%)$ & \\
\hline Serum creatinine $(\mathrm{mg} / \mathrm{dL})^{\mathrm{c}}$ & $1.0(0.81,1.39)$ & $1.0(0.8,1.57)$ & 0.97 \\
\hline Platelet count $\left(\times 10^{3} / \mu \mathrm{L}\right)^{\mathrm{c}}$ & $163(101,264)$ & $191(115,242)$ & 0.44 \\
\hline Total leucocyte count $\left(\times 10^{3} / \mu \mathrm{L}\right)^{\mathrm{c}}$ & $12.8(8.1,21.0)$ & $13.6(7.4,18.3)$ & 0.55 \\
\hline Serum $\mathrm{C}$ reactive protein $(\mathrm{mg} / \mathrm{L})$ & $132 \pm 116$ & $104 \pm 112$ & 0.41 \\
\hline \multicolumn{4}{|l|}{ Systemic inflammatory response } \\
\hline 3 of 4 SIRS criteria & $33(56 \%)$ & $27(49 \%)$ & \multirow[t]{2}{*}{0.46} \\
\hline 4 of 4 SIRS criteria & $26(44 \%)$ & $28(51 \%)$ & \\
\hline \multicolumn{4}{|l|}{ Organ dysfunction $^{\mathrm{a}}$} \\
\hline Cardiovascular & $31(52.5 \%)$ & $28(50.9 \%)$ & 0.86 \\
\hline Renal & $9(15.3 \%)$ & $8(14.5 \%)$ & 0.92 \\
\hline Respiratory & $27(45.8 \%)$ & $29(52.7 \%)$ & 0.46 \\
\hline Hematological & $10(16.9 \%)$ & $8(14.5)$ & 0.73 \\
\hline Metabolic acidosis & $8(13.6 \%)$ & $4(7.3 \%)$ & 0.27 \\
\hline Central nervous system & $7(11.9 \%)$ & $8(14.6 \%)$ & 0.67 \\
\hline \multicolumn{4}{|l|}{ Number of organs affected } \\
\hline One organ & $38(64.4 \%)$ & $36(65.5 \%)$ & \multirow[t]{4}{*}{0.91} \\
\hline Two organs & $17(28.8 \%)$ & $16(29.1 \%)$ & \\
\hline Three organs & $3(5.1 \%)$ & $3(5.5 \%)$ & \\
\hline Four organs & $1(1.7 \%)$ & 0 & \\
\hline \multicolumn{4}{|l|}{ Infection causing sepsis } \\
\hline Respiratory & 25 & 22 & \multirow[t]{6}{*}{0.79} \\
\hline Abdominal & 22 & 18 & \\
\hline Urinary tract & 4 & 4 & \\
\hline Central nervous system & 0 & 1 & \\
\hline Skin and soft tissue & 4 & 3 & \\
\hline Bloodstream infection & 4 & 7 & \\
\hline \multicolumn{4}{|l|}{ Type of infection } \\
\hline Community-acquired & $44(75 \%)$ & $42(76 \%)$ & \multirow[t]{2}{*}{0.82} \\
\hline Nosocomial & $15(25 \%)$ & $13(24 \%)$ & \\
\hline \multicolumn{4}{|l|}{ Organisms isolated } \\
\hline Gram negative organisms & 11 & 7 & \multirow[t]{3}{*}{0.66} \\
\hline Gram positive organisms & 3 & 2 & \\
\hline Candida spp. & 1 & 2 & \\
\hline APACHE II score ${ }^{\mathrm{b}}$ & $13.5 \pm 6.5$ & $13.2 \pm 5.9$ & 0.81 \\
\hline Vasopressors use & $30(50.8 \%)$ & $28(50.9 \%)$ & 0.99 \\
\hline Mechanical ventilation & $24(41 \%)$ & $24(44 \%)$ & 0.75 \\
\hline Non-invasive & 2 & 4 & \\
\hline Invasive & 22 & 20 & \\
\hline \multicolumn{4}{|l|}{ Chronic comorbid conditions ${ }^{\text {a }}$} \\
\hline Diabetes mellitus & 6 & 9 & \multirow[t]{10}{*}{0.96} \\
\hline COPD & 4 & 5 & \\
\hline Chronic liver disease & 3 & 2 & \\
\hline Tuberculosis & 4 & 4 & \\
\hline Congestive heart failure & 4 & 2 & \\
\hline HIV with AIDS & 1 & 1 & \\
\hline Chronic kidney disease & 1 & 1 & \\
\hline Stroke & 0 & 1 & \\
\hline Autoimmune vasculitis & 1 & 1 & \\
\hline No comorbid conditions & 37 & 38 & \\
\hline
\end{tabular}

a Some patients had more than one organ dysfunction or comorbid condition

b Values are mean \pm standard deviation

${ }^{c}$ Values are median (interquartile range) treated with ulinastatin $[19,23,24]$. Small randomized controlled trials have also been conducted by a single group of investigators comparing standard treatment with intravenous administration of ulinastatin in combination with alpha thymosin and found a reduction of mortality in patients with severe sepsis [25-27]. Since these studies have all used a combination of two novel agents, it is unclear whether the survival benefit seen was due to either one of these agents, or the combination. A small Korean study showed that mortality was lower in patients 
Table 2 Outcomes in patients with severe sepsis in the placebo and ulinastatin groups

\begin{tabular}{|c|c|c|c|}
\hline & Placebo group $(n=59)$ & Ulinastatin group $(n=55)$ & $p$ value \\
\hline 28-day all-cause mortality & $12(20.3 \%)$ & $4(7.3 \%)$ & $0.045^{*}$ \\
\hline \multicolumn{4}{|l|}{ New-onset organ dysfunction ${ }^{a}$} \\
\hline Cardiovascular & 9 & 4 & 0.18 \\
\hline Respiratory & 8 & 4 & 0.27 \\
\hline Hematological & 7 & 4 & 0.41 \\
\hline Hepatic & 3 & 2 & 1.0 \\
\hline Renal & 4 & 2 & 0.68 \\
\hline Central nervous system & 3 & 2 & 1.0 \\
\hline Total number of patients with new-onset organ dysfunction & 26 & 10 & 0.003 \\
\hline Duration of vasopressor use (days) & $1.7 \pm 2.1$ & $1.7 \pm 1.9$ & 0.92 \\
\hline Duration of mechanical ventilator (days) & $11.9 \pm 9.6$ & $7.5 \pm 7.5$ & 0.18 \\
\hline Ventilation-free days (till day 28) & $10.2 \pm 12.5$ & $19.4 \pm 10.6$ & 0.019 \\
\hline Length of hospital stay (days) & $24.2 \pm 7.2$ & $11.8 \pm 7.1$ & $<0.001$ \\
\hline
\end{tabular}

Table 3 Variables associated with 28-day all-cause mortality on forward stepwise multiple logistic regression analysis

\begin{tabular}{llll}
\hline Variable & Odds ratio $^{\mathrm{a}}(\mathrm{OR})$ & $95 \%$ CI of OR & $p$ value \\
\hline Renal failure & 6.37 & $1.70-23.8$ & 0.006 \\
Need for mechanical ventilation & 3.36 & $1.01-11.2$ & 0.048 \\
Treatment with ulinastatin & 0.26 & $0.07-0.95$ & 0.042 \\
\hline
\end{tabular}

Treatment with ulinastatin was independently associated with decreased mortality compared with treatment with placebo after adjusting for other baseline characteristics including age, gender, Glasgow coma scale, specific organ system failure, number of

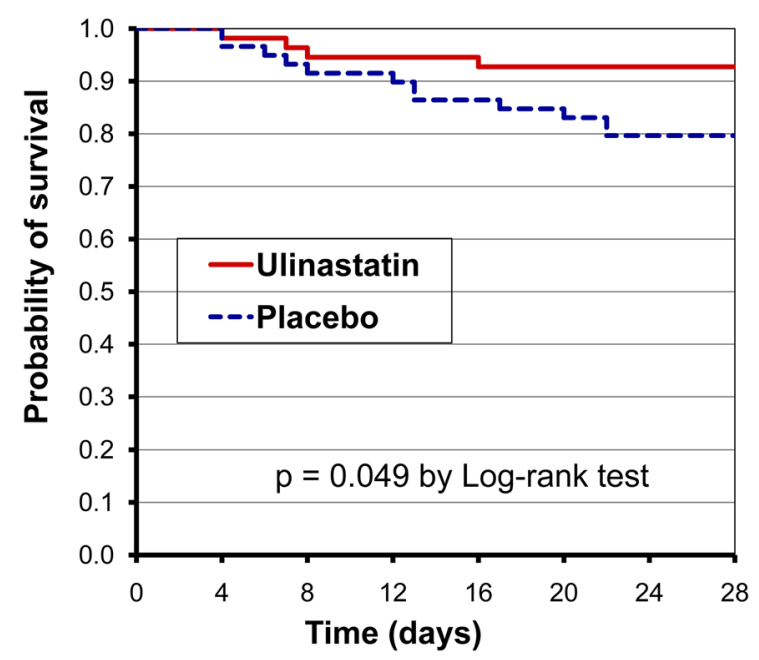

Number at risk

$\begin{array}{lllllllll}\text { Ulinastatin } & 55 & 54 & 52 & 52 & 51 & 51 & 51 & 51 \\ \text { Placebo } & 59 & 57 & 54 & 53 & 51 & 49 & 47 & 47\end{array}$

Fig. 2 Kaplan-Meier analysis of the probability of survival of patients with severe sepsis treated with ulinastatin or placebo (modified intention-to-treat cohort) organs failed, need for vasopressors and need for mechanical ventilation

${ }^{\mathrm{a}} \mathrm{OR}<1$ indicates reduced risk of death; $\mathrm{OR}>1$ indicates increased risk of death

with severe sepsis treated with ulinastatin (18.6 vs. $27 \%$ in the control group) [18]. Our results further corroborate these studies and suggest that treatment with ulinastatin may reduce mortality in severe sepsis in humans.

Highly selective serine protease inhibitors that act on only a few steps in the multipronged inflammatory response involved in the pathogenesis of sepsis like coagulation (tissue factor pathway inhibitor, activated protein $\mathrm{C}$, thrombomodulin, and antithrombin III), complement cascade ( $\mathrm{C} 1$ inhibitor), or neutrophil elastase (sivelestat) have failed to provide substantial clinical benefit in clinical trials [3-6, 21]. In contrast, ulinastatin inhibits a wide variety of pro-inflammatory serine protease enzymes including trypsin, thrombin, kallikrein, plasmin, cathepsin, neutrophil elastase, neutrophil protease-3, and coagulation factors IXa, Xa, XIa, and XIIa [7$11,28]$. Although the exact mechanism of action of ulinastatin in sepsis is not clear, it is likely that it may attenuate the inflammatory response by acting at multiple sites. In animal models of sepsis, exogenously administered ulinastatin has been shown to reduce levels of TNF$\alpha$, IL-1, IL-6, cytokine-induced neutrophil chemoattractant-1 (CINC-1), myeloperoxidase, free oxygen radicals, 
high mobility group box 1 (HMGB1), interleukin (IL)-6, interleukin-8 (IL-8), malondialdehyde, and soluble intercellular adhesion molecule-1 (sICAM-1) in serum and in organs like lung, kidney, and intestine of rats with lipopolysaccharide-induced SIRS [13-16, 28-34]. Ulinastatin also decreases phosphorylation of p38 mitogen-activated protein kinase (p38-MAPK) which in turn attenuates activation of NF- $\mathrm{KB}$ and downregulates expression of the TNF- $\alpha$ gene [33]. Studies in humans too have shown that patients with sepsis treated with intravenous administration of ulinastatin have lower serum levels of proinflammatory markers like TNF- $\alpha$, IL- $1 \beta$, IL-4, IL-6, and C-reactive protein, while levels of anti-inflammatory cytokine IL-10 was significantly higher [19, 23, 35-37]. It also reduces thrombomodulin levels and decreases endothelial dysfunction [38].

Besides reduction in 28-day all-cause mortality, ulinastatin also showed beneficial effects on some secondary end-points in the present study like new-onset organ dysfunction. Although the duration of vasopressor use was similar in the two groups, ventilator-free days were significantly higher in the ulinastatin group (19.4 vs. 10.2 days), suggesting faster recovery from severe sepsis. This also translated into a shorter mean hospital stay in the ulinastatin group.

No infusion-related adverse effects were seen in the present study. Adverse effects with ulinastatin are rare and were reported in $0.84 \%$ of patients in a Japanese study [39]. These included increased transaminases $(0.36 \%)$, eosinophilia or leucopenia $(0.16 \%)$, rash $(0.13 \%)$, gastrointestinal symptoms $(0.08 \%)$, fever $(0.02 \%)$, and local irritation at the injection site $(0.02 \%)$. These were reported in less ill patients with pancreatitis or when the drug was used prophylactically in patients undergoing endoscopic retrograde pancreatography, for which the drug is licensed in Japan. None of these effects were seen in the present study, probably because patients were too sick to complain of these symptoms.

Our study has some limitations. Firstly, although larger than previous randomized controlled studies with ulinastatin, the number of patients is relatively small, thereby limiting the power of this study. However, power of a study is more relevant for interpreting results of clinical trials that show no difference between the two treatment arms. Secondly, patients aged $>60$ years were excluded. This is because unlike in western countries, the number of elderly patients seeking care in Indian ICUs is comparatively small, and limitation of care is often opted for by the family in such cases after a few days of ICU stay owing to socio-economic and cultural reasons [40, 41]. Thirdly, the baseline APACHE II scores were low although patients had significant organ dysfunction, as has also been reported in previous studies from India [42, 43]. This is because the APACHE II score allocates more points to old age than for maximum possible physiological derangement (5 points for age 65-74 years and 6 for age $>75$ years) [44]. Moreover, septic encephalopathy is also more common in the elderly [45], and the highest possible points for any single physiological variable in the APACHE II system are assigned to the level of consciousness (APACHE II points $=15$ minus Glasgow coma score) [44]. These two factors alone could add up to 18 points per patient (6 for age and 12 for coma), accounting for high APACHE II scores in studies from western countries. These differences may limit the generalizability of our results to some extent. However, our study had some strengths too. Whereas all previous studies with ulinastatin in sepsis have been single-center studies, this was a multicenter study. Use of a doubleblind design was another strength.

In conclusion, this prospective, multicenter, randomized, double-blind, placebo-controlled pilot study in patients with severe sepsis investigated a novel therapy directed against the systemic inflammatory response. We found that 5-day treatment with intravenous administration of ulinastatin in patients with severe sepsis, when started within $48 \mathrm{~h}$ of organ dysfunction, resulted in a reduction in 28-day all-cause mortality to $7.3 \%$ from $20.3 \%$ observed in the placebo group. The reduction in mortality to $10.2 \%$ from $20.6 \%$ in the intention-to-treat population, however, was short of statistical significance $(p=0.11)$. We also found a reduction in new onset of organ dysfunction, days of mechanical ventilation, and hospital stay. A larger randomized controlled study with ulinastatin is needed to further confirm the survival benefit seen in this pilot study and also to investigate its mechanism of action in humans.

Conflicts of interest This study was funded by Bharat Serums and Vaccines Limited, India who provided the study medications, administrative assistance, and study grants to participating centers and funded the statistical analysis by an external statistician.

Dilip R. Karnad was responsible for the study design and helped in the analysis of data and preparing the manuscript. He has received consulting fees from Bharat Serum and Vaccines Limited. He has also received consulting fees from Quintiles Cardiac Safety Services and honoraria for lectures from Abbott Nutrition, India and Bharat Serums and Vaccines Limited. All other authors participated as investigators in the clinical trial and also helped in preparing and reviewing the manuscript; their institutions received grants for this clinical trial. No other conflicts of interest are declared by other authors.

Open Access This article is distributed under the terms of the Creative Commons Attribution Noncommercial License which permits any noncommercial use, distribution, and reproduction in any medium, provided the original author(s) and the source are credited. 


\section{References}

1. Angus DC, Linde-Zwirble WT, Lidicker J, Clermont G, Carcillo J, Pinsky MR (2001) Epidemiology of severe sepsis in the United States: analysis of incidence, outcome, and associated costs of care. Crit Care Med 29:1303-1310

2. Vincent JL, Rello J, Marshall J, Silva E, Anzueto A, Martin CD, Moreno R, Lipman J, Gomersall C, Sakr Y, Reinhart K (2009) International study of the prevalence of infection and outcomes of infections in intensive care units. JAMA 302:2323-2329

3. Rittirsch D, Flierl MA, Ward PA (2008) Harmful molecular mechanisms in sepsis. Nat Rev Immunol 8:776-787

4. Van der Poll T, Opal SM (2008) Hostpathogen interaction in sepsis. Lancet Infect Dis 8:32-43

5. Levi M, van der Poll T (2013) Endothelial injury in sepsis. Intensive Care Med 39:1839-1842

6. Angus D, van der Poll T (2013) Severe sepsis and septic shock. N Engl J Med 369:840-851

7. Inoue K, Takano H (2011) Urinary trypsin inhibitor, an alternative therapeutic option for inflammatory disorders. In: Nagal A (ed) Inflammatory diseases-a modern perspective. InTech, Croatia. ISBN: 978-953-307-444-3.

http://www.intechopen.com/books/ inflammatory-diseases-amodernperspective/urinary-trypsininhibitor-an-alternative-therapeuticoption-for-inflammatory-disorders. Accessed 1 June 2013

8. Sharony R, Yu PJ, Park J, Galloway AC, Mignatti P, Pintucci G (2010) Protein targets of inflammatory serine proteases and cardiovascular disease. J Inflamm (Lond) 7:45

9. Kessenbrock K, Dau T, Jenne DE (2011) Tailor-made inflammation: how neutrophil serine proteases modulate the inflammatory response. J Mol Med 89:23-28

10. Pugia MJ, Lott JA (2005)

Pathophysiology and diagnostic value of urinary trypsin inhibitors. Clin Chem Lab Med 43:1-16

11. Shigetomi H, Onogi A, Kajiwara H, Yoshida S, Furukawa N, Haruta S, Tanase Y, Kanayama S, Noguchi T, Yamada Y, Oi H, Kobayashi H (2010) Anti-inflammatory actions of serine protease inhibitors containing the Kunitz domain. Inflamm Res 59:679-687
12. Lim YP, Bendelja K, Opal SM, Siryaporn E, Hixson DC, Palardy JE (2003) Correlation between mortality and the levels of inter-alpha inhibitors in the plasma of patients with severe sepsis. J Infect Dis 188:919-926

13. Wakahara K, Kobayashi H, Yagyu T, Matsuzaki H, Kondo T, Kurita N et al (2005) Bikunin suppresses lipopolysaccharide-induced lethality through down-regulation of tumor necrosis factor- $\alpha$ and interleukin- $1 \beta$ in macrophages. J Infect Dis 191:930-938

14. Ueki M, Taie S, Chujo K, Asaga T, Iwanaga Y, Ono J, Maekawa N (2007) Urinary trypsin inhibitor reduces inflammatory response in kidney induced by lipopolysaccharide. J Biosci Bioeng 104:315-320

15. Tanaka R, Fujita M, Tsuruta R, Fujimoto K, Aki HS, Kumagai K et al (2010) Urinary trypsin inhibitor suppresses excessive generation of superoxide anion radical, systemic inflammation, oxidative stress, and endothelial injury in endotoxemic rats. Inflamm Res 59:597-606

16. Huang N, Wang F, Wang Y, Hou J, Li J, Deng X (2013) Ulinastatin improves survival of septic mice by suppressing inflammatory response and lymphocyte apoptosis. J Surg Res 182:296-302

17. Cao YZ, Tu YY, Chen X, Wang BL, Zhong YX, Liu MH (2012) Protective effect of ulinastatin against murine models of sepsis: inhibition of TNF- $\alpha$ and IL- 6 and augmentation of IL-10 and IL-13. Exp Toxicol Pathol 64:543-547

18. Moon SW, Lee SW, Hong YS, Park DW, Jang IJ, Yoon YH, Lim SI (2009) The effects of urinary trypsin inhibitor on the outcomes of severe sepsis and septic shock patients. J Korean Soc Emerg Med 20:80-85

19. Shao Y, Zhang L, Deng L, Yao H (2005) Clinical study on effects of ulinastatin on patients with systemic inflammatory response syndrome. Zhongguo Wei Zhong Bing Ji Jiu Yi Xue 17:228-230

20. Opal SM, Lim YP, Cristofaro P Artenstein AW, Kessimian N, DelSesto D, Parejo N, Palardy JE, Siryaporn E (2011) Inter-alpha inhibitor proteins: a novel therapeutic strategy for experimental anthrax infection. Shock 35:42-44

21. Antonopoulou A, GiamarellosBourboulis EJ (2011) Immunomodulation in sepsis: state of the art and future perspective. Immunotherapy 3:117-128
22. Bernard GR, Vincent JL, Laterre PF, LaRosa SP, Dhainaut JF, Lopez-

Rodriguez A et al (2001) Efficacy and safety of recombinant human activated protein $\mathrm{C}$ for severe sepsis. N Engl J Med 344:699-709

23. Wu TJ, Zhang LN, Kang CC (2013) The effect of ulinastatin on disbalance of inflammation and immune status in patients with severe sepsis. Zhonghua Wei Zhong Bing Ji Jiu Yi Xue 25:219-223

24. Yang H, Zhen XF, Li HY (2006) Clinical observation of ulinastatin in the treatment of multiorgan dysfunction syndrome. J Guangdong Coll Pharm 6:036

25. Chen H, He MY, Li YM (2009)

Treatment of patients with severe sepsis using ulinastatin and thymosin $\alpha 1$ : a prospective, randomized, controlled pilot study. Chin Med J 122:883-888

26. Li YM, Chen H, Li X, Zhou W, He MY, Chiriva-Internati M, Wachtel MS, Frezza EE (2009) A new immunomodulatory therapy for severe sepsis: ulinastatin plus thymosin alpha 1. J Intensive Care Med 24:47-53

27. Zhang Y, Chen H, Li YM, Zheng SS, Chen YG, Li LJ, Zhou L, Xie HY, Praseedom RK (2008) Thymosin alpha1- and ulinastatin-based immunomodulatory strategy for sepsis arising from intra-abdominal infection due to carbapenem-resistant bacteria. J Infect Dis 198:723-730

28. Nakatani K, Takeshita S, Tsujimoto H, Kawamura Y, Sekine I (2001) Inhibitory effect of serine protease inhibitors on neutrophil-mediated endothelial injury. J Leukoc Biol 69:241-247

29. Inoue K, Takano H, Shimada A, Yanagisawa R, Sakurai M, Yoshino S, Sato H, Yoshikawa T (2005) Urinary trypsin inhibitor protects against systemic inflammation induced by lipopolysaccharide. Mol Pharmacol 67:673-680

30. Bae HB, Jeong CW, Li M, Kim HS, Kwak SH (2012) Effects of urinary trypsin inhibitor on lipopolysaccharideinduced acute lung injury in rabbits. Inflammation 35:176-182

31. Tani T, Aoki H, Yoshioka T, Lin KJ, Kodama M (1993) Treatment of septic shock with a protease inhibitor in a canine model: a prospective, randomized, controlled trial. Crit Care Med 21:925-930

32. Inoue $\mathrm{K}$, Takano $\mathrm{H}$, Yanagisawa $\mathrm{R}$, Yoshikawa T (2008) Protective effects of urinary trypsin inhibitor on systemic inflammatory response induced by lipopolysaccharide. J Clin Biochem Nutr 43:139-142 
33. Zhang X, Liu F, Liu H, Cheng H, Wang W, Wen Q, Wang Y (2011) Urinary trypsin inhibitor attenuates

lipopolysaccharide-induced acute lung injury by blocking the activation of p38 mitogen-activated protein kinase. Inflamm Res 60:569-575

34. Massberg S, Grahl L, von Bruehl ML, Manukyan D, Pfeiler S, Goosmann C, Brinkmann V, Lorenz M, Bidzhekov K, Khandagale AB, Konrad I, Kennerknecht E, Reges K, Holdenrieder S, Braun S, Reinhardt C, Spannagl M, Preissner KT, Engelmann B (2010) Reciprocal coupling of coagulation and innate immunity via neutrophil serine proteases. Nat Med 16:887-896

35. Endo S, Inada K, Taki K, Hoshi S, Yoshida M (1990) Inhibitory effects of ulinastatin on the production of cytokines: implications for the prevention of septicemic shock. Clin Ther 12:323-326

36. Kawai S, Sakayori S, Watanabe H, Kobayashi H (1990) Usefulness of a protease inhibitor (urinastatin) in ARDS with infectious diseases. Nihon Kyobu Shikkan Gakkai Zasshi 28:843-851
37. Aibiki M, Cook JA (1997) Ulinastatin, a human trypsin inhibitor, inhibits endotoxin-induced thromboxane B2 production in human monocytes. Crit Care Med 25:430-434

38. Abe M, Ishikawa M, Soga Y, Suga H, Nakagawa T, Suzuki T (2004) Acute and high dose therapy of urinary trypsin inhibitor could inhibit vascular endothelial cell disorders in critical illness. Crit Care 8(suppl 1):199

39. Luo PF, Ren Y, Liu Y-L, Xia Z-F (2001) Ulinastatin and thymosin as immune modulators for the treatment of sepsis. Cochrane Database Syst Rev (7):CD009940. doi: 10.1002/14651858.CD009940

40. Kapadia F, Singh M, Divatia J, Vaidyanathan P, Udwadia FE, Raisinghaney SJ, Limaye HS, Karnad DR (2005) End of life limitation and withdrawal of intensive therapy at the end of life: practices in intensive care units in Mumbai, India. Crit Care Med 33:1272-1275

41. Kulkarni HS, Kulkarni KR,

Mallampalli A, Parkar SR, Karnad DR, Guntupalli KK (2011) Comparison of anxiety, depression, and post-traumatic stress symptoms in relatives of ICU patients in an American and an Indian public hospital. Indian J Crit Care Med 15:147-156
42. Panchabhai TS, Dangayach NS, Krishnan A, Kothari VM, Karnad DR (2009) Oropharyngeal cleansing with $0.2 \%$ chlorhexidine for prevention of nosocomial pneumonia in critically ill patients: an open-label randomized trial with $0.01 \%$ potassium permanganate as control. Chest 135:1150-1156

43. Todi S, Chatterjee S, Sahu S, Bhattacharyya M (2010) Epidemiology of severe sepsis in India: an update. Crit Care 14(Suppl 1):382

44. Knaus WA, Draper EA, Wagner DP, Zimmerman JE (1985) APACHE II: a severity of disease classification system. Crit Care Med 13:818-829

45. van den Boogaard M, Pickkers P, Slooter AJ, Kuiper MA, Spronk PE, van der Voort PH, van der Hoeven JG, Donders R, van Achterberg T, Schoonhoven L (2012) Development and validation of PRE-DELIRIC (PREdiction of DELIRium in ICu patients) delirium prediction model for intensive care patients: observational multicentre study. Br Med J 344:e420 